\title{
The electron-impact broadening parameters for Co III spectral lines ${ }^{\star}$
}

\author{
D. Tankosić ${ }^{1,2}$, L. Č. Popović ${ }^{1,2}$, and M. S. Dimitrijević ${ }^{1,2}$ \\ 1 Astronomical Observatory, Volgina 7, 11160 Belgrade 74, Serbia, Yugoslavia \\ 2 Isaac Newton Institute of Chile, Yugosavia Branch \\ Received 11 October 2002 / Accepted 21 November 2002

\begin{abstract}
In hot star atmospheres, the Stark broadening mechanism is the main pressure broadening mechanism. An interesting application where such a mechanism is of interest is the modeling and investigation of hot star spectra, stellar atmospheres and subphotospheric layers. Consequently, for the investigation and modeling of the Hg-Mn star and other type of hot star atmospheres, the Stark broadening parameters for Co III spectral lines may be of interest.

Here we present Stark broadening data for 20 Co III spectral lines (from $a^{6} \mathrm{D}-z^{6} \mathrm{D}^{\circ}$ and $a^{6} \mathrm{D}-z^{6} \mathrm{~F}^{0}$ Co III multiplets), as a function of temperature, calculated by using the modified semi-empirical approach. The importance of the electron-impact effect in the case of the Co III $194.98 \mathrm{~nm}$ line for several stellar atmosphere models has been tested.
\end{abstract}

Key words. atomic processes - line formation - stars: atmospheres - stars: early-type

\section{Introduction}

Spectral lines of doubly charged heavy element ions are present in hot star spectra, and they are of particular interest for modeling of CP star atmospheres. The spectral lines for singly ionized cobalt are present, e.g. in Hg-Mn star spectra (Bolcal \& Didelon 1987). The investigation of cobalt abundance in $\mathrm{Hg}$ Mn stars shows that most of the $\mathrm{Hg}-\mathrm{Mn}$ stars are largely cobaltdeficient $([\mathrm{Co} / \mathrm{H}] \leq-2 \mathrm{dex})$, except for the mildly cobalt-rich stars, $v \mathrm{Cnc}$ and $\phi \mathrm{Her}$, and the cobalt-normal stars 87 Psc and 36 Lyn (Smith \& Dworetsky 1993).

In hot star atmospheres the Stark broadening mechanism is the main pressure broadening mechanism (see e.g. Dimitrijević 1989; Popović et al. 1999, 2001). This broadening mechanism also may be important in the case of cooler stars, e.g. solar type stars, for transitions involving higher principal quantum numbers (Vince et al. 1985). An interesting application where such a mechanism is of interest is also the modeling and investigation of subphotospheric layers (Seaton 1987). Consequently, for the investigation and modeling of the $\mathrm{Hg}-\mathrm{Mn}$ star and other types of hot star atmospheres, the Stark broadening parameters for Co III spectral lines are of interest. We underline that with instruments like the Goddard High Resolution Spectrograph on the Hubble Space Telescope, good resolution spectra with line profiles of trace elements become available. Here we present the Stark broadening data for 20 Co III spectral lines calculated using the modified semi-empirical approach (Dimitrijević \& Konjević 1980; Dimitrijević \& Kršljanin 1986; Popović \& Dimitrijević 1996).

Send offprint requests to: D. Tankosić

* Table 1 is only available in electronic form at the CDS via anonymous ftp to cdsarc.u-strasbg.fr $(130.79 .128 .5)$ or via http://cdsweb.u-strasbg.fr/cgi-bin/qcat?]/A+A/399/795

\section{Theory}

According to the modified semi-empirical (MSE) approach (Dimitrijević \& Konjević 1980; Dimitrijević \& Kršljanin 1986) the electron impact full width $(F H W M)$ of an isolated ion line is given as

$$
\begin{aligned}
w_{M S E}= & N \frac{4 \pi}{3 c} \frac{\hbar^{2}}{m^{2}}\left(\frac{2 m}{\pi k T}\right)^{1 / 2} \frac{\lambda^{2}}{\sqrt{3}} \cdot\left\{\sum_{\ell_{i} \pm 1} \sum_{L_{i^{\prime}} J_{i^{\prime}}} \mathfrak{R}_{\ell_{i}, \ell_{i} \pm 1}^{2} \widetilde{g}\left(x_{\ell_{i}, \ell_{i} \pm 1}\right)\right. \\
& +\sum_{\ell_{f} \pm 1} \sum_{L_{f^{\prime}} J_{f^{\prime}}} \boldsymbol{R}_{\ell_{f}, \ell_{f} \pm 1}^{2} \widetilde{g}\left(x_{\ell_{f}, \ell_{f} \pm 1}\right)+\left(\sum_{i^{\prime}} \boldsymbol{R}_{i i^{\prime}}^{2}\right)_{\Delta n \neq 0} \\
& \left.\times g\left(x_{n_{i}, n_{i}+1}\right)+\left(\sum_{f^{\prime}} \mathfrak{R}_{f f^{\prime}}^{2}\right)_{\Delta n \neq 0} g\left(x_{n_{f}, n_{f}+1}\right)\right\}
\end{aligned}
$$

and the corresponding Stark shift as

$$
\begin{aligned}
& d=N \frac{2 \pi}{3 c} \frac{\hbar^{2}}{m^{2}}\left(\frac{2 m}{\pi k T}\right)^{1 / 2} \frac{\lambda^{2}}{\sqrt{3}} \cdot\left\{\sum_{L_{i^{\prime}} J_{i^{\prime}}} \sigma_{J_{i} J_{i^{\prime}}} \mathfrak{R}_{\ell_{i}, \ell_{i}+1}^{2} \widetilde{g}_{\mathrm{sh}}\left(x_{\ell_{i}, \ell_{i}+1}\right)\right. \\
& -\sum_{L_{i^{\prime}} J_{i^{\prime}}} \sigma_{J_{i} J_{i^{\prime}}} \mathfrak{R}_{\ell_{i}, \ell_{i}-1}^{2} \widetilde{g}_{\mathrm{sh}}\left(x_{\ell_{i}, \ell_{i}-1}\right) \sum_{L_{f^{\prime}} J_{f^{\prime}}} \sigma_{J_{f} J_{f^{\prime}}} \boldsymbol{R}_{\ell_{f}, \ell_{f}+1}^{2} \widetilde{g}_{\mathrm{sh}}\left(x_{\ell_{f}, \ell_{f}+1}\right) \\
& +\sum_{L_{f^{\prime}} J_{f^{\prime}}} \sigma_{J_{f} J_{f^{\prime}}} \boldsymbol{R}_{\ell_{f}, \ell_{f}-1}^{2} \widetilde{g}_{\mathrm{sh}}\left(x_{\ell_{f}, \ell_{f}-1}\right)+\left(\sum_{i^{\prime}} \boldsymbol{R}_{i i^{\prime}}^{2}\right)_{\Delta n \neq 0} g_{\mathrm{sh}}\left(x_{n_{i}, n_{i}+1}\right) \\
& -2 \sum_{i^{\prime}\left(\Delta E_{i^{\prime}}<0\right)} \sum_{L_{i^{\prime}} J_{i^{\prime}}} \boldsymbol{R}_{\ell_{i}, \ell_{i^{\prime}}}^{2} g_{\mathrm{sh}}\left(x_{\ell_{i}, \ell_{i^{\prime}}}\right)-\left(\sum_{f^{\prime}} \boldsymbol{R}_{f f^{\prime}}^{2}\right)_{\Delta n \neq 0} g_{\mathrm{sh}}\left(x_{n_{f}, n_{f}+1}\right) \\
& \left.+2 \sum_{f^{\prime}\left(\Delta E_{f f^{\prime}}<0\right)} \sum_{L_{f^{\prime}} J_{f^{\prime}}} \boldsymbol{R}_{\ell_{f}, \ell_{f^{\prime}}}^{2} g_{\mathrm{sh}}\left(x_{\ell_{f}, \ell_{f^{\prime}}}\right)+\sum_{k} \delta_{k}\right\}^{2)}
\end{aligned}
$$


where the initial level is denoted $i$, the final one with $f$, $\mathfrak{R}_{\ell_{k}, \ell_{k^{\prime}}}^{2}, k=i, f$ is the square of the matrix element, and

$\left(\sum_{k^{\prime}} \boldsymbol{R}_{k k^{\prime}}^{2}\right)_{\Delta n \neq 0}=\left(\frac{3 n_{k}^{*}}{2 Z}\right)^{2} \frac{1}{9}\left(n_{k}^{* 2}+3 \ell_{k}^{2}+3 \ell_{k}+11\right)$

(in the Coulomb approximation).

In Eqs. (1) and (2)

$x_{l_{k}, l_{k^{\prime}}}=\frac{E}{\Delta E_{l_{k}, l_{k^{\prime}}}}, k=i, f$

where $E=\frac{3}{2} k T$ is the electron kinetic energy and $\Delta E_{l_{k}, l_{k^{\prime}}}=$ $\left|E_{l_{k}}-E_{l_{k^{\prime}}}\right|$ is the energy difference between levels $l_{k}$ and $l_{k} \pm 1$ $(k=\mathrm{i}, \mathrm{f})$,

$x_{n_{k}, n_{k}+1} \approx \frac{E}{\Delta E_{n_{k}, n_{k}+1}}$,

where for $\Delta n \neq 0$ the energy difference between energy leves with $n_{k}$ and $n_{k}+1, \Delta E_{n_{k}, n_{k}+1}$, is estimated as $\Delta E_{n_{k}, n_{k}+1} \approx$ $2 Z^{2} E_{\mathrm{H}} / n_{k}^{* 3} \cdot n_{k}^{*}=\left[E_{\mathrm{H}} Z^{2} /\left(E_{\mathrm{ion}}-E_{k}\right)\right]^{1 / 2}$ is the effective principal quantum number, $Z$ is the residual ionic charge, for example $Z=1$ for neutral atoms and $E_{\text {ion }}$ is appropriate spectral series limit.

In Eqs. (1)-(3) $N$ and $T$ are electron density and temperature, respectively, while with $g(x)$ (Griem 1968), $\widetilde{g}(x)$ (Dimitrijević \& Konjević 1980) and $g_{\mathrm{sh}}(x)$ (Griem 1968), $\widetilde{g}_{\mathrm{sh}}(x)$ Dimitrijević \& Kršljanin 1986) are the corresponding Gaunt factors for width and shift, respectively. The factor $\sigma_{k k^{\prime}}=$ $\left(E_{k^{\prime}}-E_{k}\right) /\left|E_{k^{\prime}}-E_{k}\right|$, where $E_{k}$ and $E_{k^{\prime}}$ are the energy of the considered and its pertubing level. The sum $\sum_{k} \delta_{k}$ is different from zero only if perturbing levels strongly violating the assumed approximations exist and may be evaluated as

$\delta_{i}= \pm \mathfrak{R}_{i i^{\prime}}^{2}\left[g_{\mathrm{sh}}\left(\frac{E}{\Delta E_{i, i^{\prime}}}\right) \mp g_{\mathrm{sh}}\left(x_{n_{i}, n_{i}+1}\right)\right]$,

for the upper level, and

$\delta_{f}=\mp \boldsymbol{R}_{f f^{\prime}}^{2}\left[g_{\mathrm{sh}}\left(\frac{E}{\Delta E_{f, f^{\prime}}}\right) \mp g_{\mathrm{sh}}\left(x_{n_{f}, n_{f}+1}\right)\right]$,

for the lower level. In Eqs. (4) and (5) lower signs correspond to $\Delta E_{j j^{\prime}}<0$.

In comparison with the semiclasical perturbation approach (Sahal-Bréchot 1969a,b; Dimitrijević et al. 1991), the modified semi-empirical approach needs a considerably smaller amount of atomic data. In fact, if there are not perturbing levels strongly violating the assumed approximation, for e.g. the line width calculations, we need only the energy levels with $\Delta n=0$ and $\ell_{i f}=\ell_{i f} \pm 1$, since all perturbing levels with $\Delta n \neq 0$, needed for a full semiclassical investigation are lumped together and approximately estimated. The modified semi-empirical method is used here, since we do not have a sufficiently complete set of published experimental Co III energy levels for an adequate semiclassical treatment.

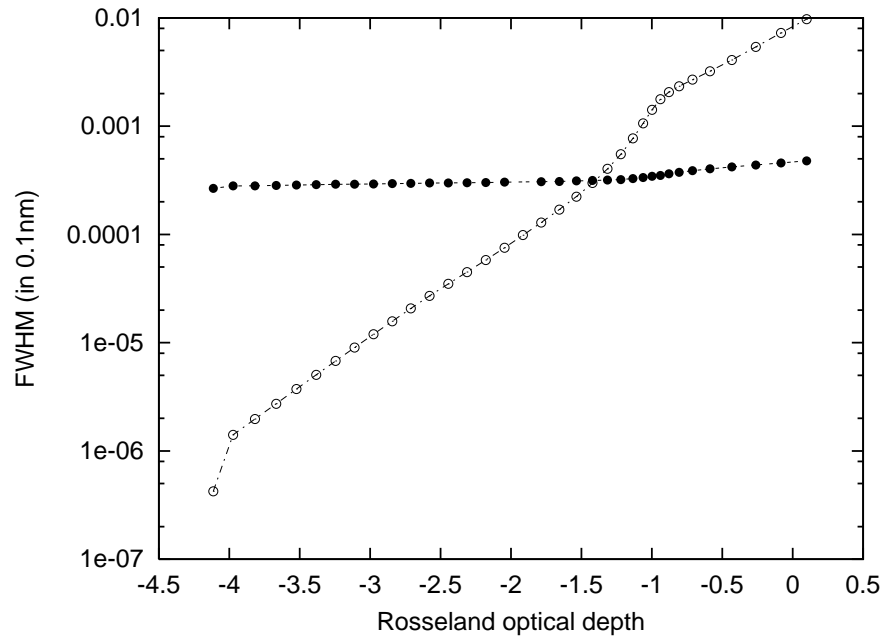

Fig. 1. Thermal Doppler (full circles) and Stark widths (open circles) for Co III $(\lambda=194.98 \mathrm{~nm})$ spectral line as functions of optical depth for an A type $\operatorname{star}\left(T_{\text {eff }}=10000 \mathrm{~K}, \log g=4\right)$.

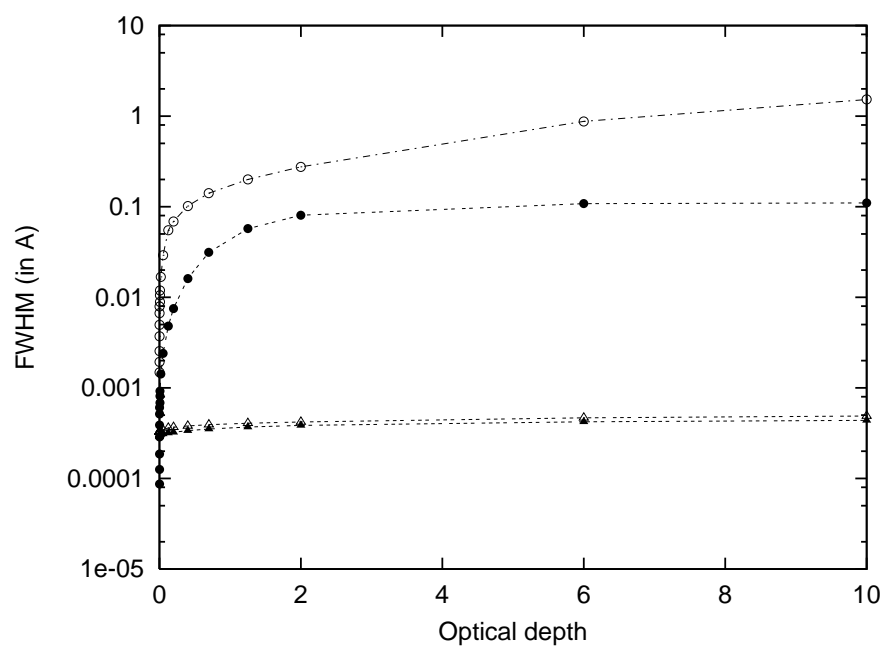

Fig. 2. Same as in Fig. 1 but for DA ( $\left.T_{\text {eff }}=10000 \mathrm{~K}, \log g=6\right)$ and DB $\left(T_{\text {eff }}=15000 \mathrm{~K}, \log g=7\right)$ white dwarfs with full circles is denoted Stark $F W H M$ for DA and with open circles for DB white dwarfs. Full triangles denote thermal Doppler $F W H M$ for DA and open triangles DA white dwarfs.

\section{Results and discussion}

The Stark broadening parameter calculations for 20 Co III spectral lines considered were performed using the modified semi-empirical approach (Dimitrijević \& Konjević 1980; Dimitrijević \& Kršljanin 1986; Popović \& Dimitrijević 1996). Oscillator strengths have been calculated using the method of Bates \& Damgaard (1949). The LS coupling approximation and the configuration mixing has been taken into account (see Popović \& Dimitrijević 1996). The Co III energy levels needed for the Stark broadening parameter calculations as well as the purity of energy levels (configuration mixing) have been taken from Sugar \& Corliss (1981). In Table 1, Stark widths and shifts for the 20 Co III spectral lines (from $a^{6} \mathrm{D}-z^{6} \mathrm{D}^{0}$ and $a^{6} \mathrm{D}-z^{6} \mathrm{~F}^{0}$ multiplets), for an electron density of $10^{23} \mathrm{~m}^{-3}$ and temperature range 10000-30000 K, are shown. We have 
calculated Stark broadening parameters for seven additional Co III lines, but the difference between lines from the same multiplet was too small and in Table 1 we only indicated that the results are practically the same. The average accuracy of the modified semi-empirical approach is $\pm 50 \%$ (Dimitrijević \& Konjević 1980).

In order to see the influence of Stark broadening mechanism for Co III spectral lines in stellar plasma conditions, we have calculated the Stark widths for the Co III $194.98 \mathrm{~nm}$ spectral line in different models of stellar atmospheres. In Figs. 1 and 2, the electron-impact and thermal Doppler widths as function of optical depth for a Kurucz's (1979) A type star $\left(T_{\text {eff }}=\right.$ $10000 \mathrm{~K}, \log g=4)$ and models of DA $\left(T_{\mathrm{eff}}=10000 \mathrm{~K}\right.$, $\log g=6)$ and DB ( $\left.T_{\text {eff }}=15000 \mathrm{~K}, \log g=7\right)$ white dwarf atmospheres (Wickramasinghe 1972) are presented. From Fig. 1, in the case of an A type star, one can observe the existence of photospheric layers where Doppler and Stark widths are comparable and where the Stark width is dominant. In the case of white dwarf atmospheres (see Fig. 2) the Stark broadening mechanism is important in all considered layers of atmospheres and in deeper atmosphere layers the Stark width is three to four orders of magnitude larger than the termal Doppler width, so that in all considered cases the Stark broadening effect should be taken into account in abundance determination, spectra synthesis and modeling of stellar plasmas.

There are no experimental data or any other theoretical calculations for comparison, so an experimental determination of Co III Stark broadening parameters is of interest.

Acknowledgements. This work is a part of the projects "Influence of collisional processes on astrophysical plasma lineshapes" and "Astrophysical Spectroscopy of Extragalactic Objects", supported by the Ministry of Science, Technologies and Development of Serbia.
The research was supported also by the Fonds zur Förderung der wissenschaftlichen Forschung (Project S7303-AST).

\section{References}

Bates, D. R., \& Damgaard, A. 1949, Trans. Roy. Soc. London, Ser. A, 242,101

Bolcal, C., \& Didelon, P. 1987, Elemental Abundance Analyses, Institut d'Astronomie de l'Université de Lausanne, Chavannesdes-Bois, Switzerland, 152

Dimitrijević, M. S. 1989, Bull. Obs. Astron. Belgrade, 140, 111

Dimitrijević, M. S., \& Konjević, N. 1980, J. Quant. Spectrosc. Radiat. Transfer, 24, 454

Dimitrijević, M. S., \& Kršljanin, V. 1986, A\&A, 165, 269

Dimitrijević, M. S., Sahal-Bréchot, S., Bommier, V. 1991, A\&AS, 89, 581

Griem, H. R. 1968, Phys. Rev., 165, 258

Griem, H. R. 1974, Spectral Line Broadening by Plasmas (Academic Press, New York)

Kurucz, R. L. 1979, ApJS, 40, 1

Popović, L. Č., \& Dimitrijević, M. S. 1996, Phys. Scr., 53, 325

Popović, L. Č., Dimitrijević, M. S., \& Ryabchikova, T. 1999, A\&A, 350, 719

Popović, L. Č., Simić, S., Milovanović, N., \& Dimitrijević, M. S. 2001, ApJS, 135, 109

Sahal-Bréchot, S. 1969a, A\&A, 1, 91

Sahal-Bréchot, S. 1969b, A\&A, 2, 322

Seaton, M. J. 1987, J. Phys. B, 20, 6363

Smith, K. C., \& Dworetsky, M. M. 1993, A\&A, 274, 335

Sugar, J., \& Corllis, C. 1981, J. Phys. Chem. Ref. Data, 10, 4

Vince, I., Dimitrijević, M. S., \& Kršljanin, V. 1985, in Progress in Stellar Line Formation Theory, ed. J. Beckman, \& L. Crivellari (Reidel, Dordrecht), 373

Wickramasinghe, D. T. 1972, Mem. R. Astron. Soc., 76, 129 